\title{
Design of Vending Machine using Finite State Machine and Visual Automata Simulator
}

\author{
Ashwag Alrehily, Ruqiah Fallatah and Vijey Thayananthan \\ Computer Science Department, \\ Faculty of Computing and Information Technology, \\ King Abdul Aziz University, \\ Jeddah 21589, Saudi Arabia
}

\begin{abstract}
Automata theory is dominating in many applications developed from the concept of finite state machine (FSM). This research will consider the design of vending machine (VM), which improves the books delivery service in the academic institution, illustrated by an application. An efficiency of VM is considered as a problem. In order to improve the efficiency, automata theory depends on the design of the FSM and visual automata simulator (VAS) are used as a method. Finally, the paper will construct FSM that uses fewer states. As a result, we will find that we can improve the efficiency and design cost of the VM.
\end{abstract}

\section{General Terms}

Automata theory and FSM are considered as our general terms. Throughout this research, VAS is also considered for graphical representation.

\section{Keywords}

Automata Theory, Vending Machine, Nondeterministic Finite State Machine, and VAS.

\section{INTRODUCTION}

Vending machines (VM) are electronic devices used to provide different products such as snakes, coffee, and tickets, etc. They are designed to be able to accept money and serve product according to the amount of money was inserted. The efficiency of VM depends on the design that uses the automata theory [1-3].

The finite-state automaton is set of all possible states and controls transitions from state to state in response to external input. It also called FSM or finite state automaton (FSA). It is a mathematical tool used to describe processes involving inputs and output [4]. Also, it is suitable for building some different kinds of software, including systems for verifying the correctness of circuits or protocols and the lexical analysis component of a compiler. It is also assisting the control unit in many physical systems including elevators, automatic traffic signals and VM. It can divide FSM into two types: Moore and Mealy state machines. The Mealy machine is also called Synchronous FSM which is a state machine that uses only input actions, and the output depends on the inputs and present state. Moore Machine also called as Asynchronous FSM is a state machine that uses only entry actions, and the output depends on the present state. The efficiency of VM directly influences with the number of states used in the design and the number of steps used in the tools employed.

In this paper, we have focused on the efficient design of book VM improved from the basic state diagram based on nondeterministic finite state machine (NFSM). Then we will simulate a model with the help of VAS tool. The proposed VM simply can provide four types of books that are: English books with 50 SR. Price, Arabic books with 50 SR. Price, Notes books with $30 \mathrm{SR}$. price and Drafts with 20 SR price. The user has to insert the right amount of special type of book and the machine will count and check if correct amount of money inserted and then serve the book directly, the user also can discard and cancel the operation. These automata designs are implemented using different simulators, VAS, which is a new tool considered for this design. Also, it's compatibility allows us to integrate with other tools.

\subsection{Scope}

This paper aim is to design VM for books, and this machine purpose is to help students in the university. It is a multi-select books machine with NFSM based. It is improved to deliver books depending on the user selection for a specific type of available books, English, Arabic, Notes, and Draft. The user then must insert amount of money type of 10, and 20 Saudi Riyal according on each book price to get the book he wants. The cancel feature was added; that means the customer can discard the ordering of product and end the buying operation. The user has to insert the amount of money equals to the price of the book he wants. The basic operations of this VM can simplify as an available type of book selection, and then ten andlor 20 Saudi Riyal money insertions, and the machine vend the book. Machine's finite state diagram will visualize and simulated using VAS, which is a tool for drawing, simulating, showing and transforming FSM. The state diagram was based on NFSM; it is was useful in our work because it enables the multi transition from one state, and this enables the user to try a multi transition to find the needed book.

\subsection{Paper organization}

The paper is designed as follow; the next part is titled with the literature review that includes the related work of previous research and some related idea of our proposed research. The third part titled with a description of VM: containing basic operations of VM, challenges and the definitions about FSM. The fourth part includes the analysis and designing process of proposed book VM, followed by fifth part of the implementation of VM model with the flowchart, results finding and implication. The last part is a conclusion of the proposed work

\section{LITERATURE REVIEW}

Regarding the efficiency of VM, many researches have been investigated the architecture of the VM. Fauziah Zainuddin [5] proposes a VM, which has the basic procedures of FSM for serving food in appropriate way. Here, the researcher used three different features in his design they are user selection state, freezer state, and steaming state.

There are a lot of researches that analyze the design of VM. In [6] they propose FPGA board VM, which provide to people 
four products, namely, Ice cream, Coca-Cola, snacks and chocolate. Using very simple steps and design, efficiency can be improved to deliver the product. The machine takes an only coin, two types of coins (one rupee and two rupee). It provides the change depending on the amount of money that the user has inserted, and there is a Cancel button to return the money to the user. Using Xilinx, State CAD tool does the VM simulation.

In [7] they propose multi-select VM, which is the Mealy model where the design of FSM is considered. The efficiency of VM can be increased using optimized coding written in VHDL language and implemented in FPGA board. This VM has four product milk, water, fruit, and sprite. It takes only coin two types of coin 5 rupee and 10 rupee. It has auto billing and cancel features. Here, Xilinx ISE simulator does the VM simulation

According to the research paper, [8], authors have described an idea of automatic ticket VM by using the sensor, RFID, and Zigbee technique. Here, they have used the automated system where people can use the smart cards that are much easy to handle during the rush times. Also, it provides secure environments because each transaction is monitored and stored for all future identification. The dedicated VM used for purchasing tickets has LCD that is the one of the intelligent features in the modern VM. Through this LCD, the people can see the details of the journey time and destination that people have planned to visit. The automated system helps us save the unnecessary cost and time. Also, it encourages us to increase the efficiency because it needs less complexity.

In these modern days, Reverse VM is very popular in different countries. It saves time and energy of the system that increase the efficiency of VM. According to [9] we can describe the Reverse VM that works when inserting the empty beverage container into it. The project of Reverse VM (RVM) is useless without the cooperation of the consumer. The best example of RVM is refund operation that is the one of the important functions in the VM.

\section{DESCRIPTIONS OF VM AND BASIC DEFINITIONS}

Here, description of VM including intelligent approach and challenges are considered. Basically, VM provides several different types of items when money inserted into it. The VMs are more practical, easy to use and accessible for user than the standard purchasing method [9], they can be found everywhere for different kind of product such as snacks and cold drink, coffee, tickets and diamonds and platinum jewelers. The efficient implementation of these machines can be in different ways by using microcontroller and FPGA board. Figure 1 shows an abstract example of VM.

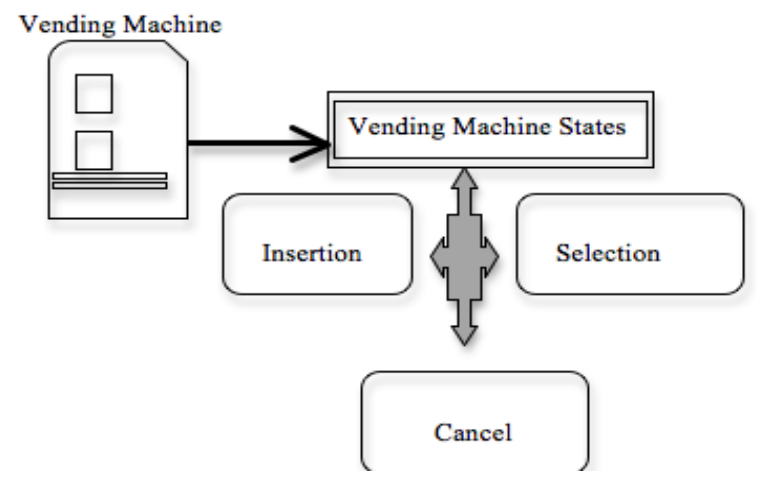

Figure 1: Basic Operations of VM.

\subsection{Basic Operation of VM}

1- The user inserts money, money counter sends to the control unit, the amount of money inserted in the VM by the user.

2- The operation buttons are active to choose the products that people like. According to the VM's internal program, VM dispenses the products when people insert the correct amount.

3- If the program is designed to return the change, VM will return the change.

4- When selected product is not available, VM will reject the service [10].

\subsection{Efficiency of Intelligent VM}

Energy efficiency is the ultimate target when intelligent VM is in commercial use. Efficiency also confirms that VM can perform their daily functions intelligently with minimum management cost. The LCD is one of the intelligent features in intelligent VM, which includes a large tabletop office with vending service machines that have enough surface area potentially to support 6 to 14 inch LCD screens.

\subsection{Challenges of an Intelligent VM}

1- Opportunities enabled by mobile phones: Accept payments and transfer operations with the using of communication technology [11].

2- Government mandates: Detail information of medical attentions, weather forecast warnings and policies are displayed through the intelligent VM.

3- Increased user interactivity: Interaction through the VM activated from peoples' smart phone which has latest features and facilities.

4- Remote management: Exchanging the necessary and secure information during the operation of VM between the vending operators and customers. Through this management, VM operators can decrease maintenance cost and increase machine availability.

5- Software flexibility: Compatibility of the features is updated with new technologies and the software.

\subsection{Definition of FSA}

Automata Theories (AT) that can be defined as an abstract of computational system machines. AT is important aspects because; it is operating a basic rule of software's digital circuits and electronic machines. Also, it is used in verifying all types of systems include finite states. FSA is a mathematical model of any computers machine designed. FSA contains inputs and outputs transferring between states. FSA can be represented by a 5 - tuple $\left(\mathrm{Q}, \sum, \delta, \mathrm{q} 0, \mathrm{~F}\right)$, where $\mathrm{Q}$ is a finite non-empty set of states. $\sum$ is a finite non-empty set of inputs called an input alphabet. $\delta$ is a function that Maps $\mathrm{Q} \times \sum$ to $\mathrm{Q}$ is called transition function. Q0 is the initial state. $\mathrm{F}$ is the set of final states [12].

$\delta(\mathrm{q}, \mathrm{w}$ a $)=\{\mathrm{p} \in \mathrm{q} \mid \exists \mathrm{r} \in \delta(\mathrm{q}, \mathrm{w}): \mathrm{p} \in \delta(\mathrm{r}, \mathrm{a})\}$

An equation (1) describe the theory of transition $\delta$ computing an accept state in such a way that every state q, which refer to the user selection and sequence of money insertion $p$, and every string $\mathrm{w}$ expressing the sequence of transition $\mathrm{r}$, and the letters used to refer to the specific type of selection [12]. 


\section{ANALYSIS AND DESIGN}

In this paper, proposed design methodology of $\mathrm{VM}$ is analyzed using state diagrams with the help of VAS tool.

\subsection{Analysis of VM}

The user can get four different kinds of books with different prices, named as English book, Arabic book, Notes, and Drafts. The prices of these books are given in Table 1 . The machine can accept ten and twenty Saudi riyal in any possible sequence, and if the user inserts any type of money different from this, the machine will not accept it. Also, if the user performs any incorrect process such as select unavailable book or inserts money less than the amount, the machine will reject the operation of providing the book.

Table 1: Books types and prices.

\begin{tabular}{|c|c|c|}
\hline Number & Price & Book name \\
\hline 1. & 50 & English book \\
\hline 2. & 50 & Arabic book \\
\hline 3. & 30 & Note \\
\hline 4. & 20 & Draft \\
\hline
\end{tabular}

There are four select (select1, select2, select3, select4) inputs are taken for selection of products. Namely SELECT1, SELECT2, SELECT3 and SELECT4. SELECT1 used for the selection of English book. Similarly SELECT2, SELECT3 and SELECT4 represents an Arabic book, Note and Draft respectively. The user inserts money of two types 10 and 20 Saudi Riyal, 1 and two inputs represent 10 and 20 Saudi riyals respectively. A cancel (c) input is used when the user wants to cancel his request. When the user cancels the request or adds more money than the desired money, the proposed VM cannot provide money for return or change. Because the money will not return the user should cancel his request only before inserted any money. The proposed VM is designed using FSM modeling, we decide to use Nondeterministic Finite Automata (NFA), because; it does not require input symbols for state transitions and is capable of transitioning to zero or more states for a given start state and input symbol. This distinguishes it from a deterministic finite automaton (DFA), in which all transitions are uniquely determined and in which an input symbol is required for all state transitions. So (NFA) enabling the user to select multiple choices from one state easily, the detail of the entire data the proposed machine is shown in Table 2.

Table 2: The detail of proposed VM input.

\begin{tabular}{|c|c|}
\hline Input & Description \\
\hline E & Select 1 for English books Transitions \\
\hline A & Select 2 for Arabic books Transitions \\
\hline N & Select 3 for Note books Transitions \\
\hline D & Select 4 for Drafts Transitions \\
\hline R & Reset $=$ back to initialize state \\
\hline
\end{tabular}

\begin{tabular}{|c|c|}
\hline $\mathrm{C}$ & End process $=$ go to cancel the state \\
\hline 1 & $10 \mathrm{SR}$ \\
\hline 2 & $20 \mathrm{SR}$ \\
\hline
\end{tabular}

\subsection{Design Methodology}

As shown in figure 2, the methodology that is a basic algorithm of VM is designed with VAS tool. The proposed book VM steps are given below.

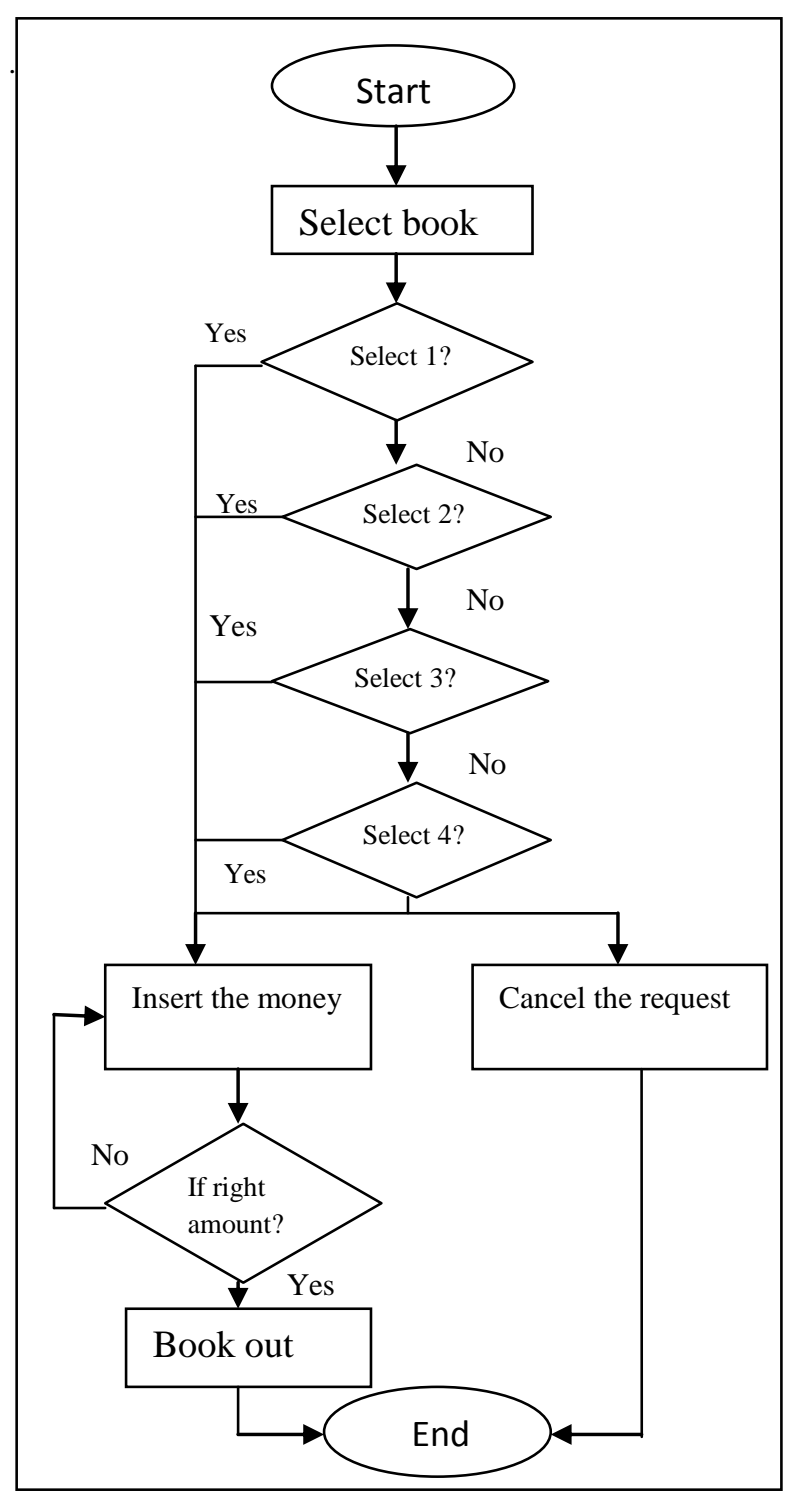

Figure 2: Flow Chart for Proposed VM

The state diagram consists of the following states (initial state, user Selection, amount of money the user inserted). The initial state is the first state of the design; the proposed machine will be ready for the users to select the book. After the initial state, the user will select the book. Then the transition will go into one of the four states (select1, select2, select3 and select 4) depended on the book the user chose. After that the machine will be waiting for the user to insert money, and the machine can accept only two types of (10 and 20) Saudi riyal. When the desired amount is inserted, the machine will go to the accepting state [13]. When the user select English book will 
go to a state then user insert a sequence of money the machine will go to specific state depending on the total amount of money as example the user enter 10, 20 and 10 Saudi Riyal, the machine will go to "10 SR " state then to "30SR" state and go to "40SR" state, At any moment, the machine has to decide whether or not to deliver the selected book or not.

\section{IMPLEMENTATION AND RESULTS}

\subsection{Simulations}

In this paper, VAS tool is used to describe design methodology of proposed book VM. It is a helpful tool for simulating, showing and transforming FSA. VAS tool was designed with a simple way to use and clear visualization of how FSA (DFA and NFA) work. It is provided ability to save the finite state diagram easily using *.fa files type. (See Figure 4) Represent the behavior of the machine for all possible sequences of money. Any state has tow circles inside each other are accepting state: As soon as the machine reaches this state, the book delivers. Thus, for four books and two types of Saudi Riyal (10 and 20), there are twenty states. The price of the Arabic book and the English book is the same. Thus; the transition from Select 2 goes to the same transition of select 1.So the state diagram only shows the 16 states excluding the five states of select 2. This is an important issue in developing proposed VM with fewer states. The machine will work much faster with using fewer states.

\subsection{Results of Simulations}

Our simulation takes string from the user; the string includes the selected book, money, and cancels if the user wants to cancel. The summation runs the string in the state diagram and as a result it accepts or rejects the entered string if the string is accepted the book delivered otherwise will not deliver the book. The next subsections are an example of accept operation.

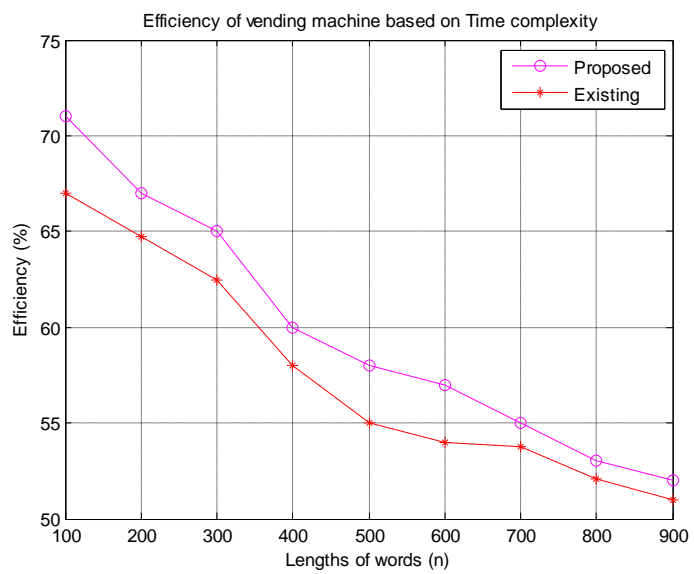

Figure 3: Comparison of efficiency

As shown in the figure 3 , the efficiency of proposed and existing design is compared. Here, lengths of words are considered to analyze the efficiency of the design based on the automata theory and VAS. During the operations, steps focus on reducibility and decidability are important because they control the complexities. When lengths of words are increased, complexity will increase with the operation time.
For all $\mathrm{n}$ and inputs of words' length $\mathrm{n}$, the execution of proposed algorithm takes at most $f(n)$ steps. Here, $f(n)$ is the time complexity of proposed algorithm. The efficiency of VM depends on which processing takes less energy to complete the operation of required feature. The Overall efficiency that includes energy saving controls the lifetime of the VM. Generally, efficiency is proportional to the power used during the operations and processing of VM. So, changes in the energy efficiency depend on the time and power used in the processing channels.

\subsection{Implementation of Accepting Operation}

The user should select a specific book and enter 10,10,10,10 and 10 for example in order to get the Arabic book. Table 3 transition details for the serving book show these transitions for a serving book using the book vending. The string is a11111b. The Final $b$ inserted refer to the end of the operation; it is only referred to the debug steps for reach accept state. After the selection of book type, the user has to insert the money, which is the amount of the specific book, and in our explanation example equal to $50 \mathrm{SR}$. At the first time, the user may insert 10SR and continue, or the user can use a single payment.

Table 3: Transition details for serving Book

\begin{tabular}{|c|c|c|}
\hline \multicolumn{3}{|c|}{ Selected string a11111b } \\
\hline Symbol & $\begin{array}{c}\text { Remaining } \\
\text { string }\end{array}$ & States \\
\hline A & $11111 \mathrm{~b}$ & {$[$ Select 2$]$, accepting = false } \\
\hline 1 & $1111 \mathrm{~b}$ & {$[10 \mathrm{SR}]$, accepting $=$ false } \\
\hline 1 & $111 \mathrm{~b}$ & {$[20 \mathrm{SR}]$, accepting $=$ false } \\
\hline 1 & $11 \mathrm{~b}$ & {$[30 \mathrm{SR}]$, accepting $=$ false } \\
\hline 1 & $1 \mathrm{~b}$ & {$[40 \mathrm{SR}]$, accepting $=$ false } \\
\hline 1 & $\mathrm{~b}$ & {$[50 \mathrm{SR}]$, accepting $=$ True } \\
\hline
\end{tabular}

Efficiency depends on the complexity that directly involves with the number of states that we use for constructing the FSA design [14]. Although time, space and other complexities are involved in the design, we use time complexity as an example. When we minimize the time complexity, we can increase the efficiency. So, we assume that randomized FSM of intelligent VM design M has time complexity $T_{c}^{M}(n)$

$$
T_{c}^{M}(n)=\max _{z \in \Sigma^{n}}\left(T_{c}^{M}(z)\right)
$$

Using (2), we can calculate $T_{c}^{M}(n)$ which, is the maximal expected running time of $\mathrm{M}$ over all words of length $\mathrm{n}$ used in the proposed FSM [15-16]. 


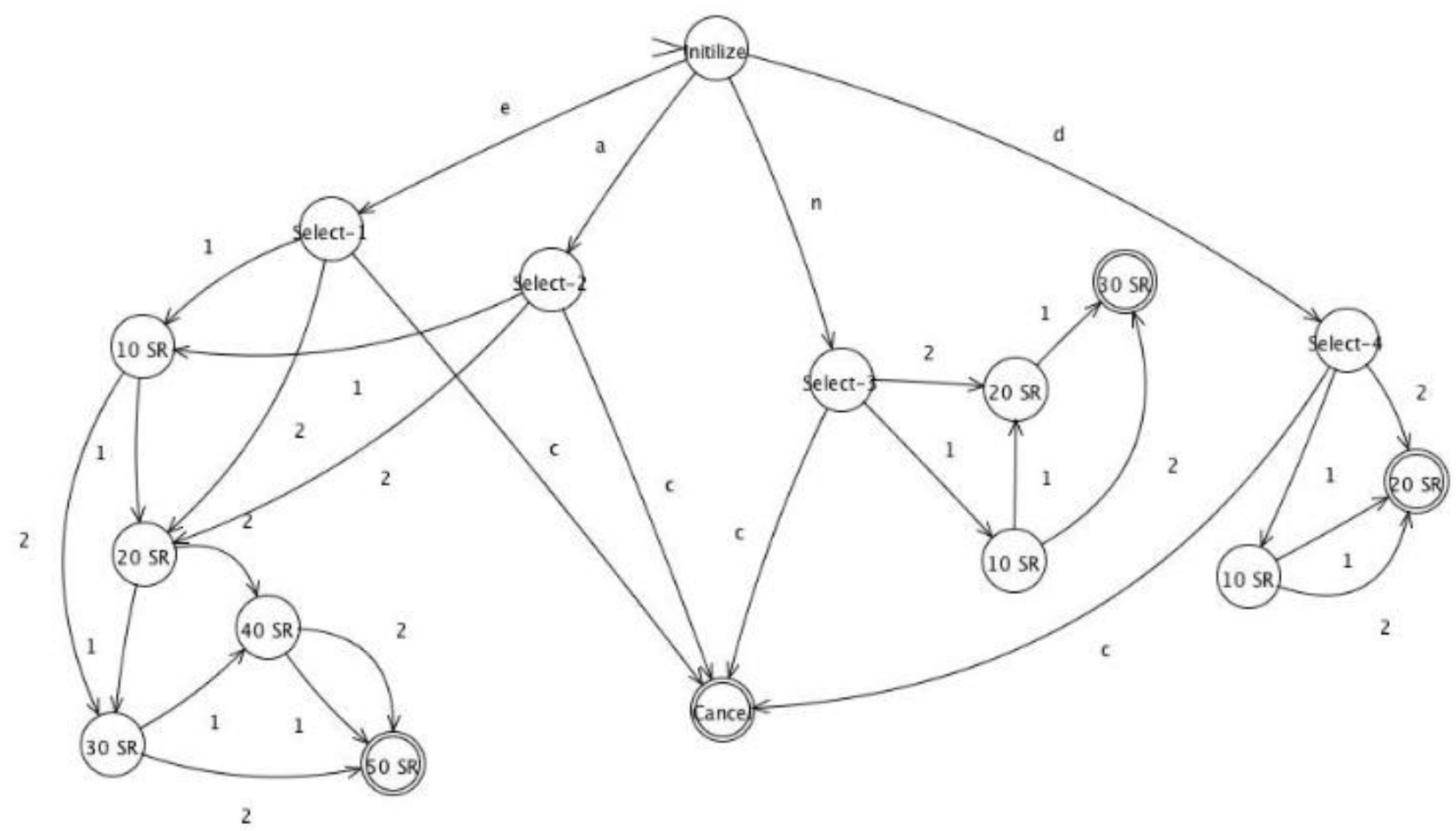

Figure 4: NFA state diagram of book VM behavior

\section{IMPLICATIONS OF RESEACH}

The studies in automata theory are abstract machines describe, as well as solving the computational problems are much easier and understandable than the other approaches. The logical understanding of input and output process depends on the study of operating virtual machines. Automata theory researches are classified as a recognizable formal language, which help the researcher to improve such application in artificial intelligent, compiler design, and other fields of the computer since that depend on computational theory.

The implication of VM depends on the number of features, which must be efficient to both customer and service providers. Increasing efficiency is dependent on the appropriate design of FSM employed in the future VM. Increasing facilities based on customer expectations, technology, time, etc.

\section{CONCLUSION}

The proposed book VM design is studied using NFSM automata theory with the help of VAS tool. Constructing FSM, which uses fewer states that enable the machine to provide fast response serving. The improvement of the efficiency and design cost was considered. Using this tool and appropriate design, the efficiency of VM can easily be enhanced for many applications. Complexity reductions that involve with the time and space increase the efficiency of the overall system used in VM.

The future work of this VM is to improving it by adds more books and scale the type of products it included according to students needs. Also, we suggest that the machine design to be able to accept ATM cards instead of paper money. Also, improving efficiency and complexity reduction by using appropriate automata machine will be possible in order to convert the VM to an intelligent approach.

\section{ACKNOWLEDGMENTS}

We are really happy that we can complete this paper successfully. We believe it cannot be completed with out an effort and co-operation among our group members and our Dr. Vijey Thayananthan. The authors also wish to thank him for his help with the solution of the theory of computation problems and applications.

\section{REFERENCES}

[1] Zhang Wen \& Zhang Xin Long, "Design and Implementation of automatic vending machine Based on the short massage payment" International Conference on Information and Communications Technology in Electrical Sciences, Neijiang, Sichuan, China.pp.978981, 2010.

[2] B. Caulfield \& M.O Mahony, "Passenger Requirements of a Public Transport Ticketing System" Proceedings of the 8th International IEEE Conference on Intelligent Transportation Systems Vienna, Austria, pp-32-37, 2005.

[3] Biplab Roy \& Biswarup Mukherjee "Design of Coffee Vending Machine using Single Electron Devices" Proceedings of 2010 International Symposium on Electronic System Design. Pp. 38-43, 2010.

[4] Hopcroft, John E. and Jeffrey D. Ullman (1979) Introduction to Automata Theory, Languages, and Computation, Reading, MA, Addison-Wesley.

[5] Fauziah Zainuddin, Norlin Mohd Ali, Roslina Mohd Sidek, Awanis Romli, Nooryati Talib \& Mohd. Izham Ibrahim, "Conceptual Modeling for Simulation: Steaming frozen Food Processing in Vending Machine" International Conference on Computer Science and Information Technology,University Malaysia Pahang, pp.145-149, 2009. 
[6] Sakshi Sharma, Ana Monga, (2011), Implementation of Reverse Vending Machine Based on FPGA, International Journal of Emerging Technologies in Computational and Applied Sciences (IJETCAS)

[7] Varkey, M. and Sunny, J. (2014). Design and Implementation of Multi Select Smart Vending Machine. International Journal of Computer Networks and Wireless Communications (IJCNWC), ISSN: 2250-3501

[8] M.Bhuvaneswari, S.Sukhumar, N.Divya, S.Kalpanadevi, N.SuthanthiraVanitha, (2013), Embedded System Based Automatic Ticket Vending Machine for Modern Transport System, International Journal of Advanced Research in Computer and Communication Engineering Vol. 2, Issue 11

[9] Ana Monga, Balwinder Singh, (2012). Finite State Machine based Vending Machine Controller with AutoBilling Features. International Journal of VLSI design \& Communication Systems (VLSICS) Vol.3, No.2.

[10] Qureshi, M., Aziz, A., Rasool, H., Ibrahim, M., Ghani, U. and Abbas, H. (2011). Design and Implementation of Vending Machine using Verilog HDL. 17.
[11] Frost \& Sullivan*, (2011), "Assessment of the Intelligent Vending Machines Market, November 2011.

[12] Pin, J. É. (2010). Mathematical foundations of automata theory. Lecture notes LIAFA, Université Paris, 7.

[13] Dash, T. and Nayak, T. (2014). Quantum Finite Automata: a Language Acceptor Model. International Journal of Advanced Research in Computer Science and Software Engineering, Volume 2(Issue 9, September 2012).

[14] John E. Hopcroft, Rajeev Motwani, Jeffrey D. Ullman, "Introduction to Automata Theory Languages and Computation". Ch-1, 27-29, $3^{\text {rd }}$ Edition Cornell University, Stanford University, California, 2001, USA.

[15] J. Holub and S. `Stekr. On parallel implementations of deterministic finite automata. In Implementation and Application of Automata, CIAA '09, pages 54-64, 2009.

[16] Vijey Thayananthan, Ashwag Alrehily and Ruqiah Fallatah, "Automata Design with Time Complexity for Intelligent Vending Machine based on Visual Automata Simulator". IEEE 17th International Conference on Computer Modelling and Simulation, UK 2015, University of Cambridge, pages 159-164. 